\title{
Numerical investigation of flow rate measure using vortex counting at low Reynolds number
}

\author{
Abdelkader Zaaraoui ${ }^{1, *}$ \\ ${ }^{1}$ Laboratoire FIMA, Université Djilali Bounaama, Route de Theniet Elhad 44225 Khemis Miliana, \\ Algérie.
}

\begin{abstract}
You The trapezoidal bluff body is a typical configuration of vortex shedding bodies. The aim of this work is to study flow behaviour over a trapezoidal cylinder at low Reynolds number. The geometry was constructed from a prototype device for measuring the volumetric flow-rate by counting vortices. Simulations were run for this geometry under steady and unsteady flow conditions using finite volume discretization. Laminar flow was investigated in this model with rigid walls and homogeneous incompressible Newtonian fluid. Calculations were performed for Reynolds number range $5 \leq \mathrm{Re} \leq 200$ and several flow parameters were documented. The present computations are in good agreement with the experimental observations and the numerical calculations by several investigators.
\end{abstract}

Keywords - bluff body, confined flow, numerical calculations, steady and unsteady flow, vortex shedding flow meter

\section{Introduction}

Flow around an obstacle has been subjected to numerous theoretical, experimental and computational investigations during several decades. The basis of flow around circular cylinders have been reported in the standard reference by Zdravkovich [1] where Reynolds numbers range were given for different regimes of flow around smooth circular cylinder in steady flow. Tian and $\mathrm{Wu}$ [2] generalized a relationship between Strouhal and Reynolds numbers by an extensive analytical and computational investigation of two-dimensional flow around regular polygons at low Reynolds number. Lee [3] studied numerically the symmetrical wake flow developments around a tapered trapezoidal cylinder. Kahawita and Wang [4] discussed briefly the influence of the trapezoidal shape on the vortex shedding and on the critical Reynolds number value. Dhiman and Hasan [5] studied the flow and heat transfer across a trapezoidal cylinder. Chung and Kang [6] reported that Strouhal number variation depends on Reynolds number and the height ratio. Pankanin [7] analyzed the various methods of investigating phenomena of the vortex flow meter and observed that the bluff body most suitable for the design of this type of device is a sharp edged trapezoidal cylinder.

\footnotetext{
* Corresponding author: a.zaaraoui@univ-dbkm.dz
} 
The flow across this kind of obstacles is complicated and still not well documented. The present work is concerned with laminar flow at low Reynolds around a trapezoidal bluff body in both steady and unsteady regimes.

\section{Methode}

\subsection{Computational geometry}

The details of geometry are reported in a paper flow measurement by vortex shedding flow meter [8]. It consists of a square-section pipe in which a two-dimensional trapezoidal cylinder is placed Fig.1-a. Mesh generation was performed using different blocking strategies. Four blocks were used to generate an o-grid type mesh around a trapezoidal cylinder. A fifth block is attached to the o-grid mesh to extend the domain in the downstream region of the flow as shown in Fig.1-b. In order to achieve mesh independent results, a mesh dependency study is done for the current case. Convergence of the grid is assessed by refining the mesh in terms of applying successively smaller elements. The grid is considered to be sufficiently fine when parameters do not differ by more than $3-5 \%$ between two succeeding grids.

a)
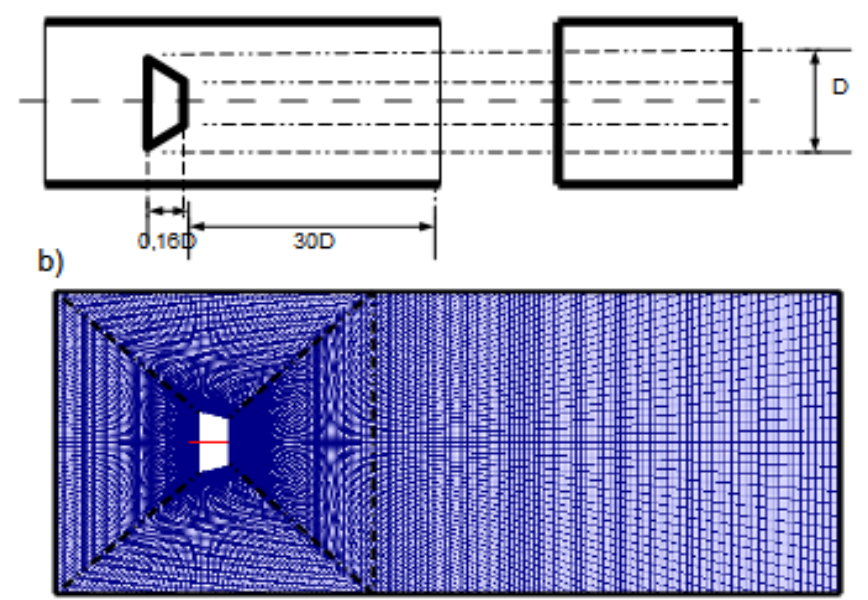

Fig. 1. Sketch of numerical grid: a) Domain dimensions, b): mesh generation

\subsection{Governing equation}

The flow was described by the dimensionless Navier-Stokes equations for unsteady laminar incompressible flow. Equations may be written as follows:

The continuity equation:

$$
\nabla \cdot U=0
$$


The momentum equation:

$$
\frac{\partial U}{\partial t}+U \cdot \nabla U=-\frac{1}{\rho} \nabla p+\frac{\mu}{\rho} \nabla^{2} U
$$

Where, $\mathrm{U}$ represents the velocity component, $p$ is the pressure, $\rho$ is the fluid density and $\mu$ is the dynamic viscosity.

\subsection{Boundary conditions}

Boundary conditions used are summarized in the table 1: The inlet condition is a uniform velocity at the inlet and a zero normal pressure gradient. The trapezoidal cylinder, upper and lower wall are considered a rigid and impermeable wall and hence the no-slip velocity and a zero normal pressure gradient are appropriate. At the domain outlet a zero normal velocity gradient and an absolute pressure of zero are prescribed.

Table 1. Boundary conditions.

\begin{tabular}{|c|c|}
\hline Inlet & $U=[100], \partial \mathrm{p} / \partial \mathrm{n}=0$ \\
\hline $\begin{array}{c}\text { Upper and lower boundaries } \\
\text { Bottom }\end{array}$ & $\mathrm{U}=[000], \partial \mathrm{p} / \partial \mathrm{n}=0$ \\
\hline Outlet & $\mathrm{U}=[000], \partial \mathrm{p} / \partial \mathrm{n}=0$ \\
\hline Trapezoidal cylinder & $\mathrm{U}=[000], \partial \mathrm{p} / \partial \mathrm{n}=0$ \\
\hline
\end{tabular}

\subsection{Numerical solution}

Solution was performed using the pisoFOAM solver [9]. It is a transient solver for incompressible laminar or turbulent flow. It is based on the Implicit with Splitting of Operators (PISO) algorithm for coupling pressure-velocity. In this algorithm: momentum equations are solved for intermediate pressure field in the predictor step without respect of continuity condition, then velocity and pressure fields are corrected in a way to fulfill both momentum and continuity equations in the two predictor steps. A standard second order finite volume discretization of Gaussian integration scheme is applied for the gradient terms using a linear interpolation scheme from cell centers to face centers. An explicit nonorthogonal correction scheme is used to compute surface normal gradients which were evaluated at cell faces. The linear corrected Gauss scheme is applied to for the Laplacian and divergence terms. The implicit first order Euler scheme is applied for the time derivative.

Convergence is assessed when the value of any parameters should not differ by more than 1-3\% between two subsequent time step, between two subsequent element size or between to subsequent grids. To ensure numerical stability in the solution, the Courant-FriedrichsLewy condition was fulfilled: 


$$
\frac{u \Delta t}{\Delta x}<1
$$

Where, $u$ is the velocity, $\Delta t$ is the time step and $\Delta x$ is the element length.

\subsection{Data analysis}

In this study, flow patterns are presented by streamlines and velocity. Also, the Q-criterion is used to identify vortices. This parameter looks at the second invariant of the velocity gradient tensor. A vortex is defined as areas where $\mathrm{Q}>0$. This means a vortex is a connected region where the antisymmetric component of the vorticity tensor, dominates over the symmetric one [10].The Finite Time Lyapunov Exponent (FTLE) was visualized to understand transport mechanism around the trapezoidal bluff body. The FTLE method is a Lagrangian approach used to compute flow map that maps particles forward/backward along their trajectories. Differentiation of the flow map will provide a measure of separation under the action of the flow [11]. For example, a recirculation region can be delimited from the overall flow by lines or surfaces in the flow on which separation is locally maximal.

\section{Results and discussions}

The Reynolds number is defined as the ration of inertial to viscous forces. It is the most decisive non-dimensional parameter for describing the flow pattern around the trapezoidal bluff body. As it is shown in the Fig.2, for very low values $(\operatorname{Re} \approx 10)$, fluid particles from the upstream of the flow were divided to two regions flowing symmetrically around upper and lower part of the trapezoidal cylinder. These particles reach together the back of the bluff body without separation. When the Reynolds number is increased $(\operatorname{Re} \approx 50)$, a small separation region was formed behind the trapezoidal cylinder which lead to the formation of twin vortices. The separation region did not extend downstream and the two eddies of opposite sign was not shed into the flow. At $\operatorname{Re}=100$ or 150 , the twin vortices elongate and a harmonic oscillations is observed in the wake region in which the flow is still laminar. Eddies start to shed alternatively from the upper and lower part of the bluff body and form the so-called Karman vortex street. In order to observe the influence of the Reynolds number on the Strouhal number, we performed a number of simulations under unstable conditions by varying the Reynolds number between 80 and 200. The number of Strouhal (St) is a number Dimension describing the oscillating circulation mechanisms, defined as follows:

$$
\mathrm{St}=\frac{\mathrm{F} \cdot D}{\mathrm{v}}
$$

\section{$F$ : Frequency of occurrence of vortices.}

$D$ : Length of the large base of the trapezium.

$\mathrm{v}$ : The flow velocity in the upstream boundary condition.

$$
\mathrm{R}_{\mathrm{e}}=\frac{v \cdot D}{v}
$$




\section{$v:$ The kinematic viscosity}

En écoulement confiné le débit de fluide est donné par :

$$
q v=v \cdot S
$$

$S$ : The cross-section of the test tunnel.

$q_{v}$ : Volume flow rate.

Table 2. Résults.

\begin{tabular}{|c|c|c|c|c|}
\hline$R_{e}$ & 80 & 100 & 150 & 200 \\
\hline$S_{t}$ & 0,136 & 0,119 & 0,088 & 0,052 \\
\hline$F\left(H_{z}\right)$ & 0,484 & 0,529 & 0,588 & 0,975 \\
\hline$q_{v}\left(\mathrm{~m}^{3} / \mathrm{s}\right)$ & 0,045 & 0,083 & 0,125 & 0,166 \\
\hline
\end{tabular}

The volumetric flow can only be measured from a laminar flow with Von Karman instability. The characteristic of the flow meter is given by the frequency of detachment of the vortices as a function of the flow rate (Tab 2). 

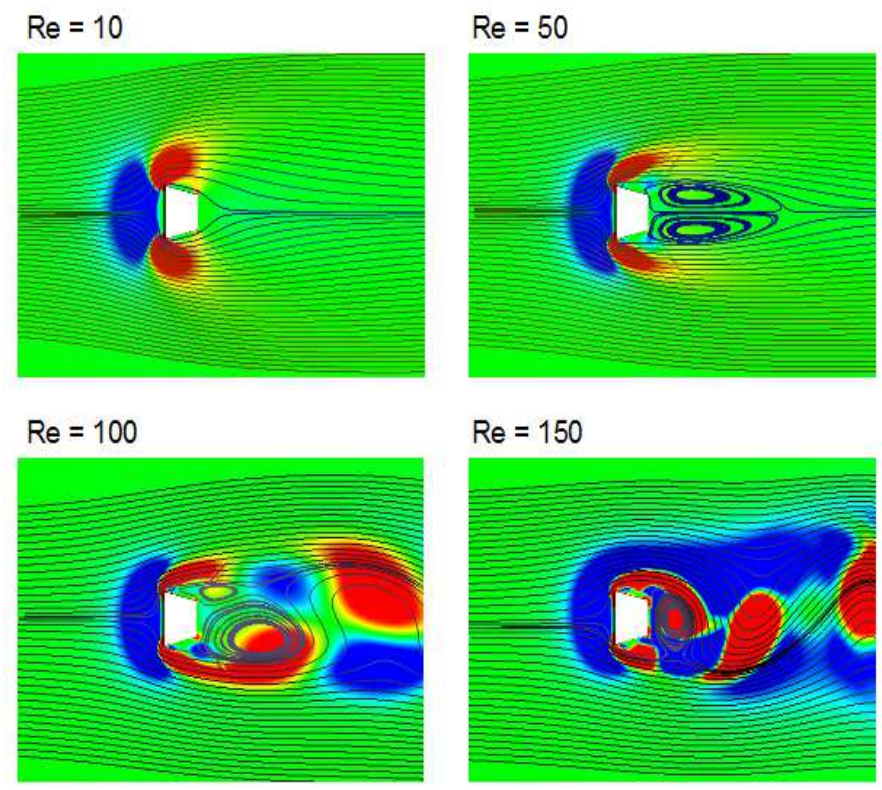

Fig. 2. Streamline for flow around the trapezoidal cylinder projected on the Q-criterion results.

As it is shown in the Fig.3, flow separates at trailing edge and forms a recirculation region which consists of two symmetric vortices. Size of recirculation zone increases with an increase of Reynolds number and by reaching the critical Reynolds number, Von Kármán Vortex Street with periodic vortex shedding happens as a result of high positive pressure gradients. Vortex shedding causes fluctuation of pressure distribution on the body surface.

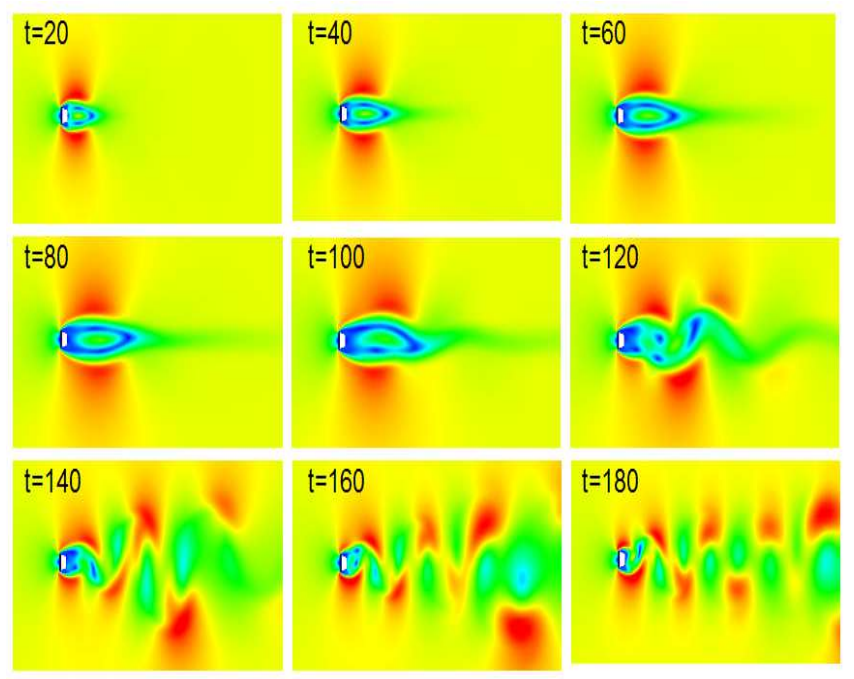

Fig. 3. Temporal evolution of the Velocity field around the trapezoidal cylinder. 
a)

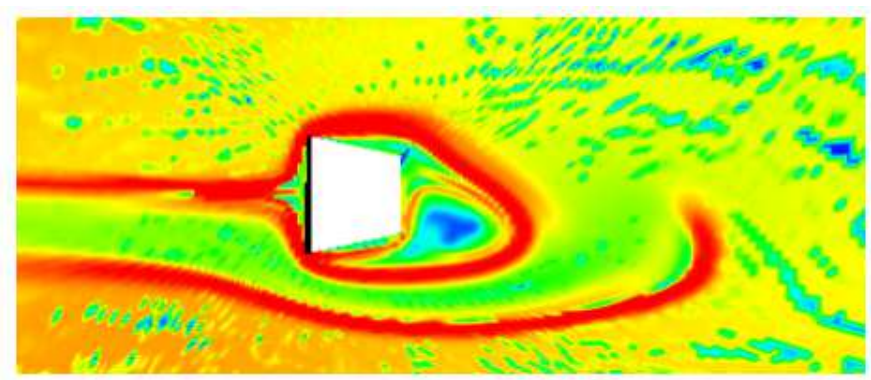

b)

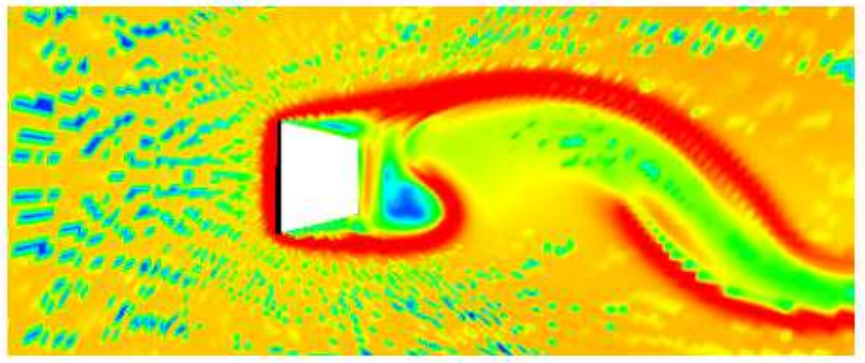

Fig. 4. FTLE contours: a) Repelling transport barriers, b) Attracting transport barriers.

Lagrangian analysis defines the boundaries of the coherent structures. It distinguishes the boundaries of all neigh boring vortices in the near cylinder region. Fig.4-a shows the contours FTLE determined in time. The flow fields with boundaries forming lobes or the fluid particle are trapped near the trapezoidal bluff body. Gradually, as the flow develops, the lobe and trapped particles are projected downstream and on the side of the obstacle is what is observed in Fig.4-b. The contours FTLE the rear of the obstacle to visualize the vortices in the wake. the fluid transport boundaries as defined by the FTLE drive the particles in the recirculation region through a jet that forms between the two LCS. The two large structures formed behind the obstacle in the wake trapezoidal form the topology of LCS, alternating closed structures. Particles entrained in the two structures downstream and particles attracted to the time backward, donations aligned in the wake.

\section{Conclusions}

In the present study, the two dimensional laminar flow around a trapezoidal bluff body was investigated at low Reynolds. For our configuration study for a confined flow, there are two flow regimes for low Reynolds numbers, stable with two different topologies of flows adhesive or unstuck and unstable with the appearance of the first instability which is the Von Karman. For the stable flow in the adhesive regime the current lines stay adhere to the obstacle while for unstuck regime two symmetric vortices stay just adhere behind the obstacle. Regarding the unstable flow, there is instability of Von Karman which appears. The detachment frequency of vortices must be determined having regard to the link with a flow measurement. The obtained results are in perfect agreement with numerical and experimental previous studies. The simulations are underway to determine the exact value of the critical Reynolds number characterizing the transition between the two flow regimes. 


\section{Acknowledgment}

The preferred Author would like to express his appreciation for the Laboratory of Industrial Fluids, Measurements and Applications at Djilali Bounaama University for providing the computing facilities that this research heavily depends upon.

\section{References}

1. M.M. Zdravkovich, flow around circular cylinder, vol. 1: Fundamentals, Oxford Scientific Publisher, (1997).

2. Z.W.Tian, Z.N.Wu, a study of two-dimensional flow past regular polygons via conformal mapping, Journal of Fluid Mechanics, 628, 121-154, (2009).

3. T.S. Lee, early stages of an impulsively started unsteady laminar flow past tapered trapezoidal cylinders, International journal for numerical methods in fluids, 26(10), 1181-1203, (1998).

4. R. Kahawita, P. Wang, numerical simulation of the wake flow behind trapezoidal bluff bodies, Computers \& fluids, 31(1), 99-112, (2002).

5. A. Dhiman, M. Hasan, flow and heat transfer over a trapezoidal cylinder: steady and unsteady regimes. Asia Pacific Journal of Chemical Engineering, 8(3), 433-446, (2013).

6. Y.J, Chung, S.H. Kang, laminar vortex shedding from a trapezoidal cylinder with different height ratios, Physics of Fluids (1994-present), 12(5), 1251-1254, (2000).

7. G.L. Pankanin, The vortex flow meter: various methods of investigating phenomena, Meas. Sci. Technol. 16, R1-R16, (2005).

8. A. Zaaraoui, F. Ravelet, F. Margnat, S. Khelladi, high accuracy volume flow rate measurement using vortex counting, Flow Measurement and Instrumentation, 33, 138144, (2013).

9. OpenCFD. 2009. OpenFOAM User Guide, 1.7.0. 203p.

10. J.C.R. Hunt, A.A. Wray, P. Moin, eddies, stream, and convergence zones in turbulent flows. Center for Turbulence Research Report CTR-S88, pp. 193-208B, (1998).

11. S.C. Shadden, F. Lekien, J. E, Marsden, definition and properties of Lagrangian coherent structures from finite-time Lyapunov exponents in two-dimensional aperiodic flows, Physica D 212, 271-304, (2005). 